\title{
Fuzzy Control System for Autonomous Navigation and Parking of Thymio II Mobile Robots
}

\author{
Fatma Boufera, Fatima Debbat, Francesco Mondada, and M. Fayçal Khelfi
}

\begin{abstract}
This paper proposed a fuzzy controller for the autonomous navigation problem of robotic systems in a dynamic and uncertain environment. In particular, we are interested in determining the robot motion to reach the target while ensuring their own safety and that of different agents that surround it. To achieve these goals, we have adopted a fuzzy controller for navigation and avoidance obstacle, taking into account the changing nature of the environment. The approach has been tested and validated on a Thymio II robots set. As application field, we have chosen a parking problem.
\end{abstract}

Index Terms-Mobile robot, navigation autonomous, fuzzy logic, Thymio II robot.

\section{INTRODUCTION}

Obstacle avoidance is an essential component to achieve successful navigation [1]. Several research works have been reported in this area. Several trajectory tracking and path following algorithms have been proposed to steer the mobile robot along a path to a desired goal in order to prevent collisions with obstacles. Many researches turned their attention to the obstacle avoidance problem developing interesting real-time behavior for mobile robots in unknown environment. The most well know are, the potential field method, vector field histogram and the method of deformable virtual zone. The first one was introduced by [2] imagines the virtual forces acting on the robot. This method assumes that the robot is driven by virtual forces that attract it towards the goal, or reject it away from the obstacles. The actual path is determined by the resultant of these virtual forces, the second method is introduced in [3] which corresponds to local occupancy grid, constructed from the sensors of the robot; this method was improved in [4], landmark learning [5], edge detection, graph-based methods [6], Limit-cycles method [7] and many others. However, relatively few of them are suitable for real-time and embedded applications on very low-cost systems. Among them, the methods that use artificial intelligence approaches as:-Neural Network [8]: this approach is applied to determine the optimal neural networks structure for realtime obstacle avoidance task. In [9], the paper proposes a

Manuscript received December 7, 2013; revised March 24, 2014.

F. Boufera and Fatima Debbat are with the Mathematics and Computer Science Department, University of Mascara, Mascara, Algeria (e-mail: fboufera@yahoo.fr, debbat_fati@yahoo.fr).

F. Mondada is with EPFL, Lausanne, Robotic Systems Laboratory (email: francesco.mondada@epfl.ch).

M. F. Khelfi is with Laboratory of Researches in Industrial Computing \& Networks, Faculty of Exact and Applied Sciences, University of Oran, Algeria (e-mail: mf_khelfi@yahoo.fr). neural network that uses Qlearning reinforcement technique for solving the problem of obstacle avoidance. -Multi Agent System: In [10], a new local collision avoidance algorithm between multiple robots for real-time navigation is presented. This algorithm is based on multi agent system and quadratic optimization method for a collision free navigation and to compute the motion of each robot. Hybrid Neural Network Genetic Algorithm: [11] used a hybrid neural net- work, genetic algorithm and local search method for solving the problem of finding the optimal collision free path in complex environments for mobile robot. -Particle Swarm Optimization: In [12], the author proposed two extensions of Particle Swarm Optimization (PSO) and Darwinian Particle Swarm Optimization (DPSO) to solve the problem of obstacle avoidance for multi robot.

All these methods require to be implemented on robots with a sufficiently complex functional structure. The robot must possess capabilities of perception, decision, evaluation of the action and a relatively high computational power and/or memory.

This paper mainly deals the navigation control and obstacle avoidance. Our objective is to develop a simple and reactive obstacle avoidance tool that can be implemented on an extremely compact such as the Thymio II [13]. In this context, we propose an intelligent and fast fuzzy controller system for navigating in real-time. The fuzzy logic is certainly one of the most adopted approaches in industry. It addresses such applications perfectly as it resembles human decision making with an ability to generate precise solutions from certain or approximate information. It fills an important gap in engineering design methods left vacant by purely mathematical approaches (e.g. linear control design), and purely logic-based approaches (e.g. expert systems) in system design. The advantage of using fuzzy logic for navigation is that it allows for the easy combination of various behaviors outputs through a command fusion process. The navigation system in this case consists of two behaviors an obstacle avoidance behavior and a goal seeking behavior [14], [15].

A set of experimentations is realized to demonstrate the feasibility of this approach for navigation, avoiding static and dynamic obstacles (other robots) and aggregation (in our case, parking in the edges of environment) for Thymio II mobile robots.

Besides this introduction, the structure of the paper is as follows: Section II gives the specifications of the robot platform and its functioning under ASEBA tool. Section III presents the description of fuzzy logic controller. Section IV shows different experimentations for testing the proposed approach. Conclusions and perspective are given in Section V. 


\section{Thymio II AND AsEba Presentation}

The system consists of a set of ThymioII mobile robots navigating in a dynamic and uncertain environment. The robots must explore the environment and avoid static and dynamic obstacles (other robots).

\section{A. Thymio II Robot}

Thymio II is an affordable educational robot $(<100 \$)$. It provides three main features: a large amount of low-cost sensors and actuators, a specific interactivity based on light and touch, aimed at increasing the understanding of the robot functionalities and a very efficient programming environment based on Aseba. Its interactivity is based on several functionalities: capacitive touch buttons, color of the body (full RGB spectrum) and LED associated with each robot functionality. This robot is representative of low-cost mass-produced systems (Fig. 1): A control method applied on the Thymio II robot can be deployed at very low cost: $<2 \$$ for the processor, $<1 \$$ for each proximity sensor, $<1 \$$ per motor.

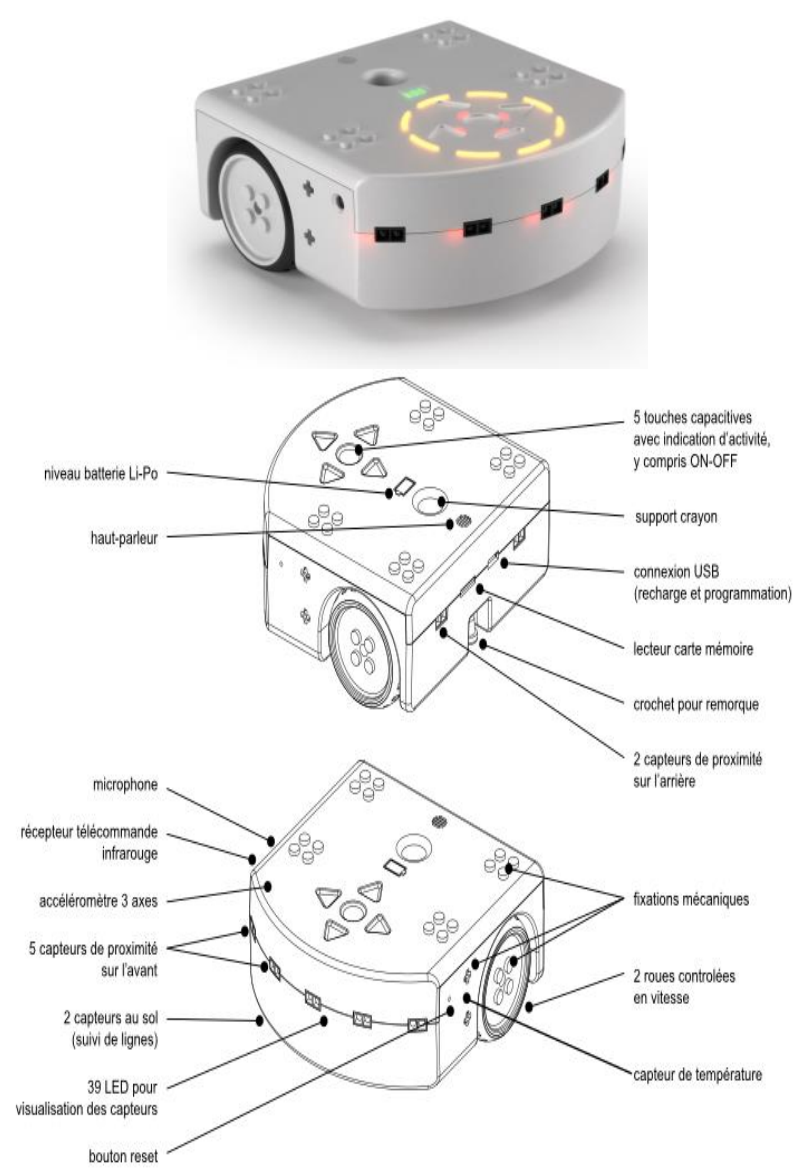

Fig. 1. The Thymio II robot platform and its structural component.

\section{B. ASEBA Environment}

Aseba is a set of tools which allow novices to program robots easily and efficiently. It is event based architecture for real-time distributed control of mobile robots. It targets integrated multi-processor robots or groups of singleprocessor units, real or simulated. The core of Aseba is a lightweight virtual machine tiny enough to run even on microcontrollers. With Aseba, we program robots in a userfriendly scripting language using a cosy integrated development environment.

\section{NAVIGATION PROPOSED APPROACH}

In order to avoid static and dynamic obstacles and permit parking in the edges of the environment by the robot Thymio II, we proposed and developed a reactive system control of navigation using fuzzy logic (Fig. 2). The robot reacts according to the changes in environment. The fuzzy system determines the two wheels speed of ThymioII robot so that it avoids obstacle and go to the reach.

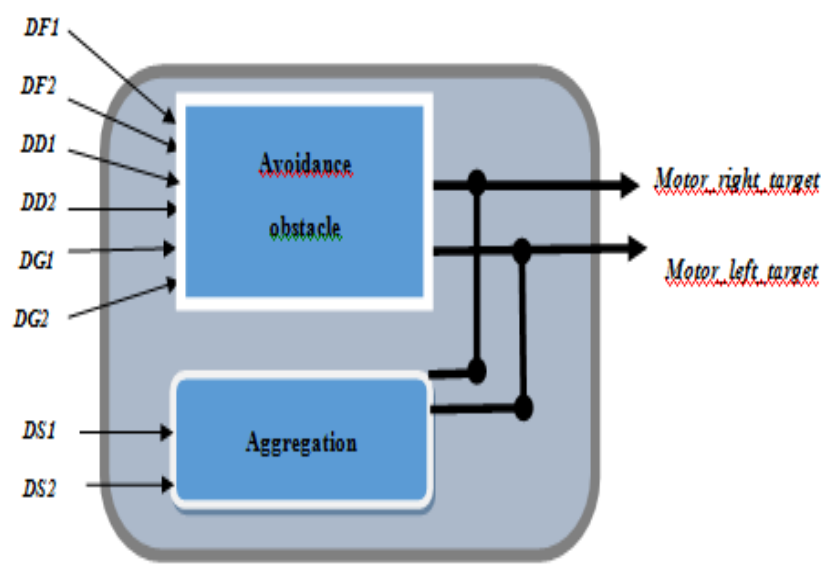

Fig. 2. ThymioII robot navigation controller.

The fuzzy control system is composed of two modules based on inputs sensory of the ThymioII robot:

- Avoidance obstacle module

- Aggregation module

A fuzzy system that determines the speed of right and left wheels: motor_right_target, motor_left_target based on the distance of the obstacles around the robot measured by the sensors: C0, C7, C6, C5, C1, C2 and values given by the two sensors DS1 and DS2 (See Table I).

TABLE I: THE MEASURED DISTANCES OBSTACLES-ROBOT
\begin{tabular}{|c|c|c|}
\hline $\begin{array}{c}\text { Obstacle } \\
\text { direction }\end{array}$ & Sensors & Distances given \\
\hline \multirow{2}{*}{ Frontal obstacle } & $\mathrm{C}_{0}$ & DF1 \\
\cline { 2 - 3 } & $\mathrm{C}_{7}$ & DF2 \\
\hline \multirow{2}{*}{ Left Obstacle } & $\mathrm{C}_{6}$ & DD1 \\
\cline { 2 - 3 } & $\mathrm{C}_{5}$ & DD2 \\
\hline \multirow{2}{*}{ Right Obstacle } & $\mathrm{C}_{1}$ & DG1 \\
\cline { 2 - 3 } & $\mathrm{C}_{2}$ & DG2 \\
\hline
\end{tabular}

\section{A. Avoidance Obstacle Module}

In the next section, we describe different stages of the fuzzy control for avoidance obstacle (Fuzzification, fuzzy inference and Deffyzzification).

\section{1) Inputs fuzzification}

We first proceed to the fuzzification of the distances: DF1, DF2, DG1, DG2, DD1, and DD2. The same fuzzy model used for the different measured distances (Fig. 3). These distances are represented by proxy_horizontal variable which is calculated by Aseba tool.The membership functions of these distances near and far have been fixed after several tests and experiments.

The prox_horizontal variable is calculated by Aseba tool. 


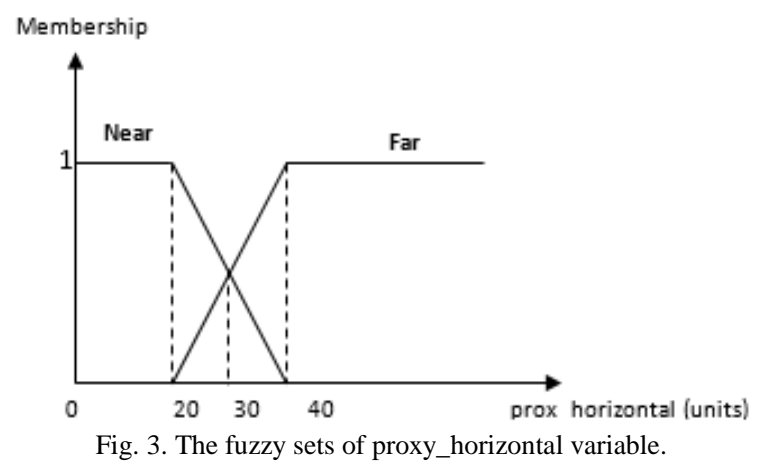

\section{2) Outputs fuzzyfication}

The speed fuzzification of the right and left wheels: motor_right_target and motor_left_target is presented in Fig. 4.

The membership functions for this variable are: Dacc (deceleration), Zacc (no speed change) and Acce (acceleration).

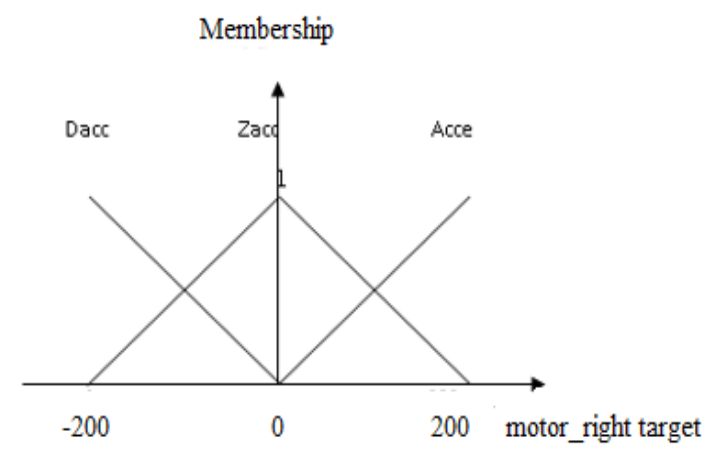

Fig. 4. Fuzzy sets of motor_right_target and motor_left_target variable.

\section{B. Fuzzy Inference}

This step presents the inference rules elaboration to determine the robot behavior according to its intrinsic parameters. It will be seen later that the number of potential rules of reaction increases directly with the number of labels of the variables.

According to the membership degree of the different distances measured by the robot sensors, each values combination of these input variables, a command presented by the outputs variables (motor_right_target and motor_left_target) is associated with it.

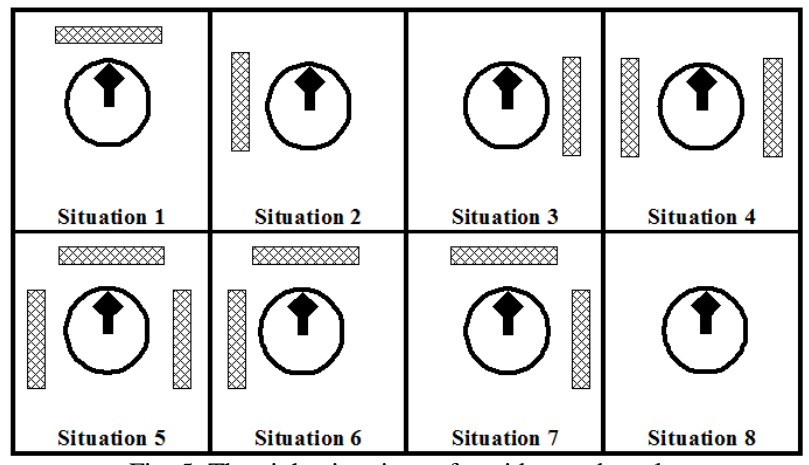

Fig. 5. The eight situations of avoidance obstacle.

The inference rules definition are based on the following situations (see Fig. 5 ) which shows the different cases of avoidance obstacles that the robot can undergo during its navigation.
Each situation is defined by a set of inference rules depending on the distances of obstacles-robot. As example, we give the set rules of situation 1: «Front close".

The robot stops and performs randomly one of the following maneuvering (Table II):

- Deviation in a right

- Deviation in a left

- A half-rotation

TABLE II: RULE INFERENCE OF SITUATION 1 "FRONT-ClOSE".

\begin{tabular}{|c|c|c|c|c|c|}
\hline \multirow{7}{*}{ If } & $\begin{array}{c}\text { Distance } \\
\text { sensor }\end{array}$ & $\begin{array}{l}\text { Fuzzy } \\
\text { Value }\end{array}$ & \multirow{7}{*}{ Then } & \multicolumn{2}{|l|}{ Action } \\
\hline & DF1 & Near & & \multirow{2}{*}{\multicolumn{2}{|c|}{ Deviation in right }} \\
\hline & DF2 & Near & & & \\
\hline & DD1 & Far & & \multirow{2}{*}{ Motor_left_target } & \multirow{2}{*}{200} \\
\hline & DD2 & Far & & & \\
\hline & DG1 & Far & & \multirow{2}{*}{ Motor_right_target } & \multirow{2}{*}{-200} \\
\hline & DG2 & Far & & & \\
\hline
\end{tabular}

\section{Defuzzification}

In this section, we present a defuzzification process used in our fuzzy system. The defuzzification step allows the transformation of the output fuzzy values into the corresponding physical values. We opted for the defuzzification method called "average weighted method". This choice is usually conditioned by a compromise between implementation ease and computing performance [16].

\section{Aggregation Module}

The objective of this module is to stop the robot when it is on the environment edges. To define these edges, we used another color (black) different in the light intensity with the inside area color (white) where the ground sensors of Thymio robot can detect it easily (Fig. 6).

The variables measured by these soil sensors are:

- prox.ground.ambiant: ambient 6light intensity on the ground, varies between 0 (no light) and 1023 (maximum light).

- prox.ground.reflected: quantity of light received while the sensor emits infrared, ranging from 0 (no reflection) and 1023 (high light).

- prox.ground.delta: difference between reflected light and ambient light, related to the distance and color of the soil.

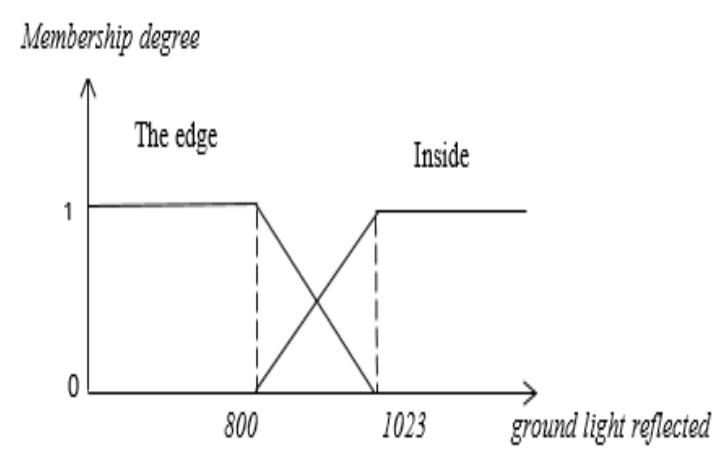

Fig. 6. Fuzzy sets of ground light reflected.

The variation of the light reflected from the ground is described by two subsets: at edges and Inside. 


\section{Simulation AND RESUlts}

In this section, we evaluate the proposed approach efficiency by a set of experimentations on Thymio II robots group (Fig. 7).

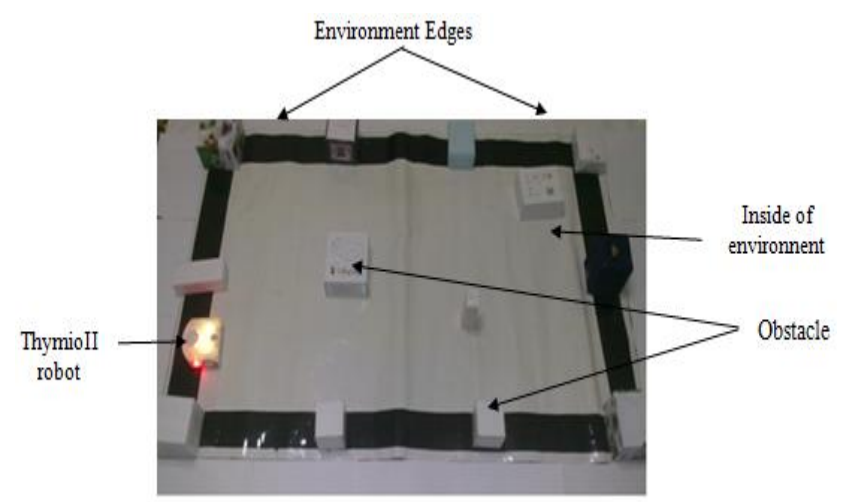

Fig. 7. Experiment environment for Thymio II robot.

The experimentation scenes are timed and filmed by several cameras. In Fig. 8-Fig. 10, we show several cases of environments simples and complexes to validate the proposed approach. The bold line represents the trajectory of the robot.

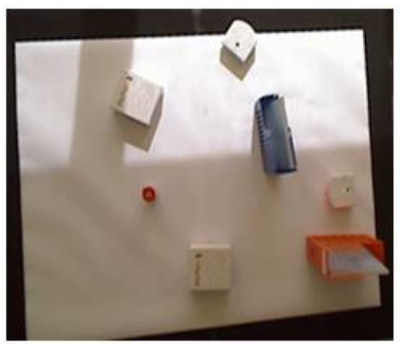

(a) First position

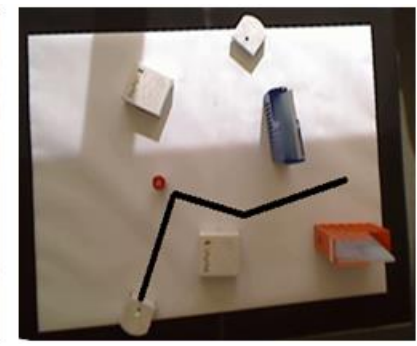

(b) Final position
Fig. 8. Navigation and avoidance obstacle in the environment 1.

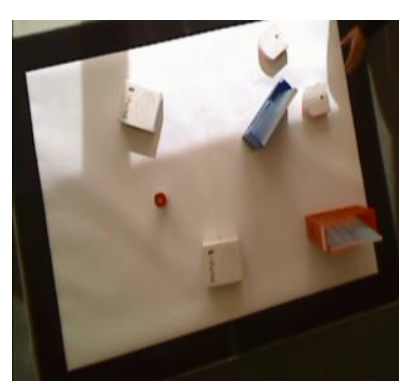

(a) First position

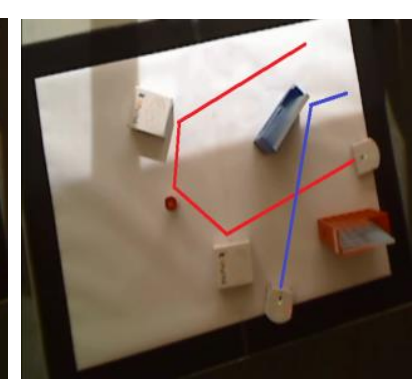

(b) Final position
Fig. 9. Navigation and avoidance obstacle in the environment 2.

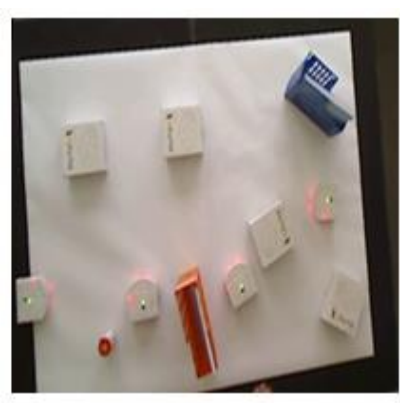

(a) First position

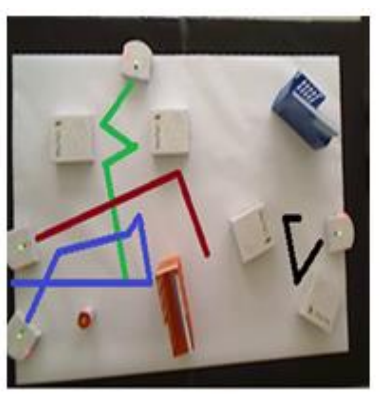

(b) Final position
Fig. 10. Navigation and avoidance obstacle in the environment 3.

In these various cases of test we can see that the proposed approach is safe and efficient.
Even if the environment is complex and dynamic (several obstacles and active robots), the ThymioII robot is able to avoiding different static and dynamic obstacles encountered with a flexible navigation and stop at the environment edges.

\section{CONCLUSION}

In this article, a proposed solution has been presented to the problem of navigation and obstacle avoidance by developing a fuzzy navigation controller dedicated to Thymio II robots under Aseba environment. The experimental results are satisfactory and validate the proposed approach. The robot navigates autonomously and safe despite the complexity of the environment.

\section{ACKNOWLEDGMENT}

The research presented in this paper was partially supported by the Swiss National Science Foundation through the National Centre of Competence in Research (NCCR) Robotics.

\section{REFERENCES}

[1] L. Adouane and N. LeFort-Piat, "Hybrid behavioural control architecture for the cooperation of minimalist mobile robots," in Proc. the International Conference on Robotics and Automation ICRA04, 2004, pp. 3735-3740.

[2] O. Khatib, "Real time obstacle avoidance for manipulators and mobile robot," International Journal Robotics Research, vol. 5, no. 1, pp. $90-80,1986$.

[3] Y. Kren and J. Borensrein, "Potential field methods and their inherent limitations for mobile robot navigation," in Proc. IEEE Conference on Robotics and Automation, 1991, pp. 1398-1404.

[4] B. Ulrich, "Reliable obstacle avoidance for fast mobile robots," in Proc. IEEE Int. Conf. on Robotics and Automation. Leuven, 1998, Belgium.

[5] J. A. Meyer and D. Filliat, "Map-based navigation in mobile robots a review of map- learning and path-planning strategies," Journal of Cognitive Systems Research, vol. 4, no. 4, pp. 283-317, 2003.

[6] L. Adouane, "Orbital obstacle avoidance algorithm for reliable and on-line mobile robot navigation," in Proc. the in 9th Conference on Autonomous Robot Systems and Competitions, 2009.

[7] L. Adouane, A. Benzerrouk, and P. Martinet, "Mobile robot navigation in cluttered environment using reactive elliptic trajectories," in Proc. the 18th IFAC World Congress, Milano-Italy, 2011.

[8] T. Hoang and T. Nguyen, "Obstacle avoidance for power wheelchair using Bayesian neural network," in Proc. the 29th Annual International, Conference of the IEEE EMBS, C, 2007, Lyon, France.

[9] M. Duguleana, F. Barbuceanua, A. Teirelbarb, and G. Mogana, "Obstacle avoidance of redundant manipulators using neural networks based reinforcement learning," Robotics and ComputerIntegrated Manufacturing, vol. 28, issue 2, pp. 132-146, 2012.

[10] S. Guy, J. Chhugani, C. Kim, N. Satish, M. Lin, D. Manocha, and P. Dubey, "Clear footpath: highly parallel collision avoidance for multiagent simulation," in Proc. Eurographics/ ACM SIGGRAPH Symposium on Computer Animation, 2009.

[11] A. Hosseinzadeh and H. Izadkhah, "Evolutionary approach for mobile robot path planning in complex environment," International Journal of Computer Science Issues, vol. 7, issue 4, 2010.

[12] M. S. Couceiro, R. P. Rocha, and N. M. F. Ferreira, "A novel multirobot exploration approach based on particle swarm optimization algorithm safety," presented at IEEE International Symposium on Security, and Rescue Robotics, 2011.

[13] S. Magnenat, F. Riedo, M. Bonani, and F. Mondada, "A programming workshop using the robot 'Thymio II': the effect on the understanding by children," presented at the IEEE International Workshop on Advanced Robotics and its Social Impacts, Munich, Germany, 2012.

[14] Cherroun. L, R. Mechgoug, and M. Boumehraz. "Path following behavior for an autonomous mobile robot using fuzzy logic and neural networks," Courrier Du Savoir, no. 12, pp. 63-70, October 2011. 
[15] P. Melin, O. Mendoza, and O. Castillo, "Face recognition with an improved interval type-2 fuzzy logic sugeno integral and modular neural networks," IEEE Transactions on Systems, Man, and Cybernetics, vol. 41, no. 5, pp. 1001-1012, 2011.

[16] P. S. Lee and L. L.Wang, "Collision avoidance by fuzzy logic control for automated guided vehicle navigation," Journal of Robotics System, vol. 11 , no. 8, pp. 743-760, 1994

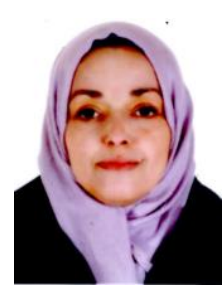

Fatma Boufera received the engineer degree in soft engineering and Ms. Degree in industrial computing from Oran University Computer Science Department, Algeria, in 2000 and 2007, respectively. She is currently working toward the Ph.D. degree in Oran University, Algeria. She is currently a research member at Mascara University Institute of Sciences and Technology, Algeria. Her research interests include mobile robotics and intelligence artificial.

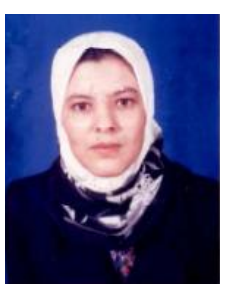

Fatima Debbat received the engineer degree in instrumentation from the Sciences and Technology University, Oran, Algeria in 1999 and the M.Tech. degree in telecommunication from Spatial Technical National Center, Arzew, Algeria in 2002. She received $\mathrm{PhD}$ degree in telecommunication in 2007 from Tlemcen University, Algeria. She is currently a research member of the Telecommunication Laboratory at Tlemcen University. Her main research interests include telecommunication.

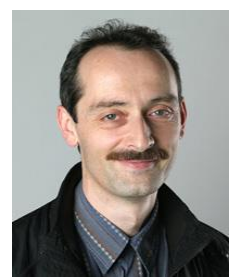

Francesco Mondada received his M.Sc. in microengineering in 1991 and his doctoral degree in 1997 at EPFL. During his thesis he co-founded the company K-Team, being both CEO and president of the company for about 5 years. He is one of the three main developers of the Khepera robot, considered as a standard in bio-inspired robotics and mentioned in thousands of publications. Fully back in research in 2000 and after a short period at CALTECH, he directed the design of several well-known robotic platforms, such as the sbot, the marXbot and the e-puck and the Thymio II robots, together reaching more than thousand mentions/citations in scientific papers. He is an author of more than 100 publications in the field of bio-inspired robotics and system level robot design. He is a co-editor of several international conference proceedings. In November 2005 he received the prestigious "Swiss Latsis University Prize," for his contributions to bio-inspired robotics. In 2011 he has been awarded with the Credit Suisse Award for Best Teaching as best teacher at EPFL. He has been appointed to be professor at EPFL in 2013. His interests include the development of innovative mechatronic solutions for mobile and modular robots, the creation of know-how for future embedded applications, and making robot platforms more accessible for education, research, and industrial development.

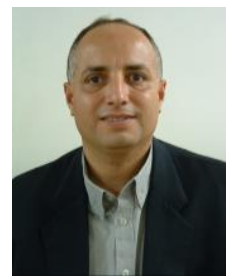

M. Fayçal Khelfi received Ph.D. degree in automatic control from Nancy University, France, in 1995. He is currently a professor at the Computer Science Department, Faculty of Exact and Applied Sciences, University of Oran, Algeria. He is also a research member at the Laboratory of Research in Industrial Computing and Networks. His main research interests include automatic control, industrial computing, robotics and networks. 